\title{
System Integration and Packaging of a Terahertz Photodetector at W-Band
}

Dong, Yunfeng; Olvera, Anuar d. J. Fernandez; Morales, Alvaro; Aller, Mario Mendez; Preu, Sascha; Zhurbenko, Vitaliy; Hanberg, Peter J.; Okonkwo, Chigo; Tafur Monroy, Idelfonso ; Johansen, Tom Keinicke

Published in:

IEEE Transactions on Components, Packaging and Manufacturing Technology

Link to article, DOI:

10.1109/TCPMT.2019.2928053

Publication date:

2019

Document Version

Peer reviewed version

Link back to DTU Orbit

Citation (APA):

Dong, Y., Olvera, A. D. J. F., Morales, A., Aller, M. M., Preu, S., Zhurbenko, V., Hanberg, P. J., Okonkwo, C., Tafur Monroy, I., \& Johansen, T. K. (2019). System Integration and Packaging of a Terahertz Photodetector at W-Band. IEEE Transactions on Components, Packaging and Manufacturing Technology, 9(8), 1486-1494. https://doi.org/10.1109/TCPMT.2019.2928053

\section{General rights}

Copyright and moral rights for the publications made accessible in the public portal are retained by the authors and/or other copyright owners and it is a condition of accessing publications that users recognise and abide by the legal requirements associated with these rights.

- Users may download and print one copy of any publication from the public portal for the purpose of private study or research.

- You may not further distribute the material or use it for any profit-making activity or commercial gain

- You may freely distribute the URL identifying the publication in the public portal 


\title{
System Integration and Packaging of a Terahertz Photodetector at W-Band
}

\author{
Yunfeng Dong, Student Member, IEEE, Anuar d. J. Fernandez Olvera, Alvaro Morales, Mario Méndez Aller, \\ Sascha Preu, Vitaliy Zhurbenko, Member, IEEE, Peter J. Hanberg, Chigo Okonkwo, Senior Member, IEEE, \\ Idelfonso Tafur Monroy, Senior Member, IEEE, and Tom K. Johansen, Member, IEEE,
}

\begin{abstract}
This paper presents the system integration and packaging of a photodetector at $\mathrm{W}$-band $(75-110 \mathrm{GHz})$ for terahertz (THz) communications. The ErAs:In(AI)GaAs photoconductor and its feeding network based on semi-insulating indium phosphide (InP) substrate are introduced. The design of the bias-tee at W-band is described and the effect of parasitic modes is discussed. Besides, the transition using E-plane probe between a W-band rectangular waveguide (WR-10) and a coplanar waveguide (CPW) is illustrated. The bias-tee as well as the E-plane probe transition are based on high-resistivity silicon (Si) substrate where wire bonding bridges are added on the top following the CPWs in order to restrict parasitic modes. The integration approach and the packaging structure are addressed. The proposed bias-tee and the E-plane probe transition including the WR-10 rectangular waveguide are fabricated, integrated, and measured. The measurement is carried out on-wafer in a back-to-back configuration and the results are presented. The assembly of the fully-packaged photodetector is demonstrated and a THz heterodyne communication system is implemented which validates the proposed system integration and packaging approach of the photodetector at W-band.
\end{abstract}

Index Terms-Bias-Tee, coplanar waveguide (CPW), E-plane probe, integration, packaging, photodetector, terahertz (THz), rectangular waveguide, wire bonding.

\section{INTRODUCTION}

$\mathbf{W}$ ITH the fast development of lightwave systems and the increasing demand for high-speed data transmissions through not only optical fibers over a long distance but also wireless links to mobile terminals [1]-[4], terahertz $(\mathrm{THz})$ photodetectors as one of the principal devices used in communication systems are under intensive study. As the optoelectronic devices that can convert signals from optical domain

Manuscript received Month DD, 2019; revised Month DD, 2019; accepted Month DD, 2019. Date of publication Month DD, 2019; date of current version Month DD, 2019. This work was supported by the European Commission funded project CELTA (Contract No. 675683).

Y. Dong, V. Zhurbenko, and T. K. Johansen are with the Electromagnetic Systems Group, Department of Electrical Engineering, Technical University of Denmark, 2800 Kgs. Lyngby, Denmark (e-mail: yundon/vz/tkj@elektro.dtu.dk).

P. J. Hanberg is with Danchip, Technical University of Denmark, $2800 \mathrm{Kgs}$. Lyngby, Denmark (e-mail: jehan@danchip.dtu.dk).

A. d. J. Fernandez Olvera, M. Méndez Aller, and S. Preu, are with the Terahertz Devices and Systems Group, Department of Electrical Engineering and Information Technology, Technische Universität Darmstadt, 64283 Darmstadt, Germany (e-mail: fernandez/aller/preu@imp.tu-darmstadt.de).

A. Morales, C. Okonkwo, and I. T. Monroy are with the Institute for Photonic Integration, Technische Universiteit Eindhoven, 5600 MB Eindhoven, Netherlands (e-mail: a.morales.vicente/cokonkwo/i.tafur.monroy@tue.nl).

Color versions of one or more of the figures in this paper are available online at http://ieeexplore.ieee.org.

Digital Object Identifier 10.1109/TCPMT.2019.xxxxxxx

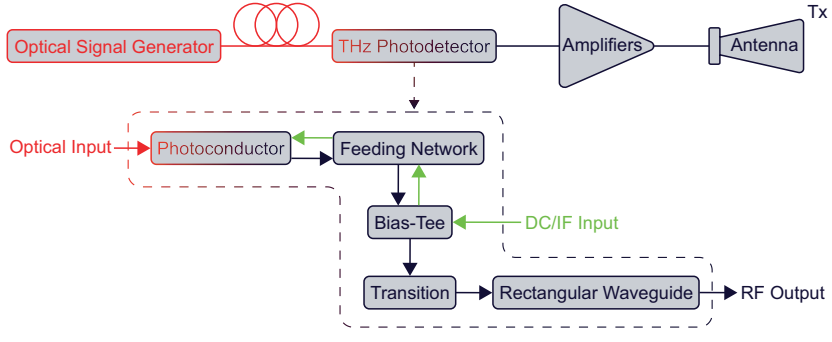

Fig. 1. System diagram of the THz photodetector as a part of the transmitter used for wireless data transmissions at W-band.

to electrical domain, photodiodes as well as photoconductors operating at $\mathrm{THz}$ frequencies have been pushed to achieve larger bandwidths [5]-[9]. Though both components can be implemented into photodetectors, they are based on different electro-optic effects which result in different characteristics. By adding an undoped region in a semiconductor junction, the photodiodes work as a rectifier converting light photons into an alternating current (AC). For the photoconductors, with a direct current (DC) bias its resistance is related to the number of free carriers in the photoconductive material generated by the light photons. At millimeter-wave and $\mathrm{THz}$ frequencies, not only the components but also the strategies for system integration and packaging are being challenged and efforts have been put to improve the performances [10]-[15]. Fig. 1 shows the system diagram of the $\mathrm{THz}$ photodetector as a part of the transmitter (Tx) used for wireless data transmissions at W-band. Instead of directly using a log-periodic antenna with a silicon lens [16], the photoconductor is integrated with other components and packaged inside a rectangular waveguide structure which allows the photodetector being cascaded with the amplifiers as well as the horn antenna in the system.

In $\mathrm{THz}$ communication systems, as one of the possible methods, a laser can be used as the light source and the data is modulated to the optical carrier by using a Mach-Zehnder modulator (MZM). After that, the modulated optical signal is transmitted to the photodetector through an optical fiber which needs to be aligned accurately with the photoconductor. The photoconductor is attached to a feeding network and the radio frequency (RF) signal is generated by the photoconductor based on the optical input. Alternatively, depending on the modulation scheme of the communication system, not only the DC bias but also the intermediate frequency (IF) signal might have to be provided to the photoconductor. As a consequence, a bias-tee is connected to the feeding network and chip-level 
connections between them are required. The bias-tee is a three-port component based on planar transmission lines where the RF signal is transmitted freely towards to the connected component while the DC bias and IF signal can only be guided to the photoconductor [17]. Since a rectangular waveguide serves as the interface of the packaging structure, a transition based on planar transmission lines is inserted for guiding the $\mathrm{RF}$ signal from the bias-tee to the rectangular waveguide. Besides, the rectangular waveguide isolates the DC bias as well as the IF signal so that the DC block can be avoided in the packaging structure. The organization of the paper is as follows. In Section II, the designs of feeding network, bias-tee, and transition are described. The fabrication, integration, and packaging of the components are addressed. In Section III, the assembly of the fully-packaged photodetector is demonstrated. Besides, a $\mathrm{THz}$ heterodyne communication system is implemented and the measurement results are presented. Conclusions are finally drawn in Section IV.

\section{PACKAGING CONSIDERATIONS}

\section{A. Feeding Network}

In order to guide the RF signal generated by the photoconductor to the bias-tee and the IF signal together with the DC bias to the photoconductor, a transmission line feed is implemented. A coplanar waveguide (CPW) is used due to its simple planar structure, tunable dimension, and low dispersion loss in a wide frequency range. Fig. 2 shows the proposed CPW feed based on a semi-insulating indium phosphide (InP) substrate, which has a thickness of $350 \mu \mathrm{m}$. The dielectric constant $\left(\epsilon_{r}\right)$ and dissipation factor $(\tan \delta)$ of the InP substrate are 12.56 and $2 \mathrm{e}-4$, respectively [18]. Gold is used as the material of the conductor layer and its thickness is $200 \mathrm{~nm}$. The metal carrier under the InP substrate also works as the bottom ground plane. The CPW is designed to have a characteristic impedance of $50 \Omega$ which supports not only DC bias but also low-loss transmissions up to $110 \mathrm{GHz}$. The width of the signal trace is $50 \mu \mathrm{m}$ and the width of the ground traces is $300 \mu \mathrm{m}$ with a gap of $50 \mu \mathrm{m}$. For aligning the photoconductor, the width of the signal trace is tapered to $8 \mu \mathrm{m}$ at the end while the ground traces are merged into a ground pad. The distance between the signal trace and the ground pad is $6 \mu \mathrm{m}$. Besides, wire bonding bridges on the top of the substrate are implemented along the CPW which are used for restricting parasitic modes. The height of the wire bonding bridges is $50 \mu \mathrm{m}$ and they are placed $160 \mu \mathrm{m}$ from each other. In the simulation, lumped ports are used as the excitation scheme and the photoconductor is represented by a port which is assigned to a surface located in the gap between the signal trace and the ground pad.

Fig. 3 shows the simulation results of the CPW feed based on InP substrate with wire bonding bridges in comparison with the situation when wire bonding bridges are removed. Due to the simple through line structure of the CPW feed and a length of $680 \mu \mathrm{m}$, similar performances are achieved and no visible notch is observed up to $110 \mathrm{GHz}$. In both cases, the return loss remains better than $17 \mathrm{~dB}$ and the insertion loss is less than $0.5 \mathrm{~dB}$.

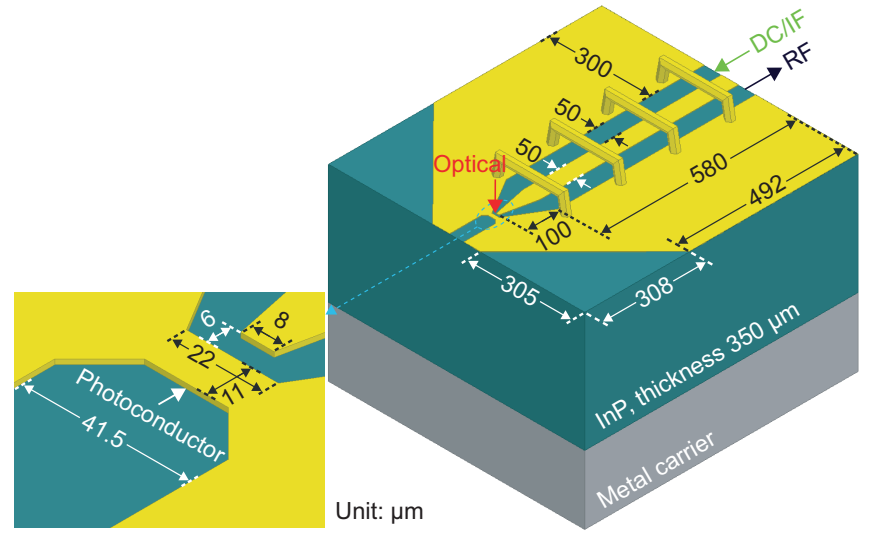

Fig. 2. CPW feed based on InP substrate for the photoconductor.

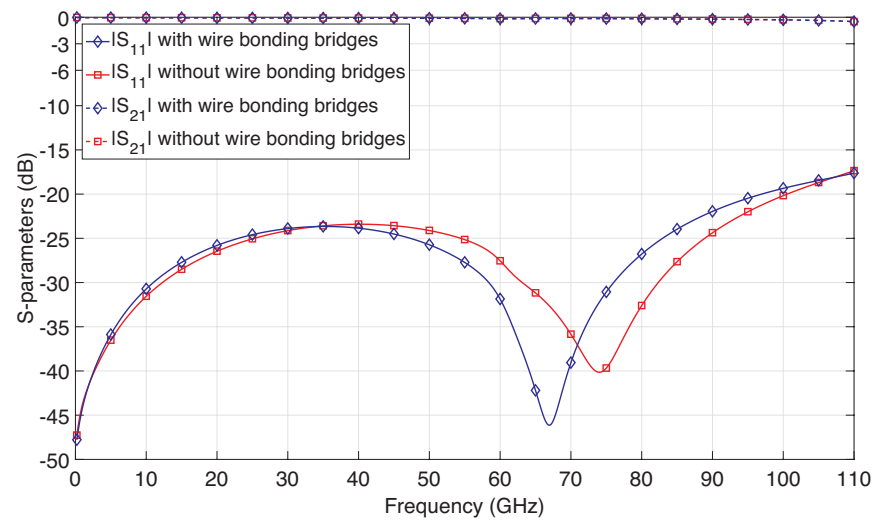

Fig. 3. Simulation results of the CPW feed based on InP substrate.

\section{B. Bias-Tee}

As is illustrated in Fig. 4, a bias-tee is designed for guiding the RF signal from the connected feeding network to the following transition while bringing the DC bias as well as the IF signal to the photoconductor at the same time. Since the bias-tee is a three-port component, except for supporting lowloss transmissions at W-band, it is also important to provide a good isolation for the RF signal so that it will be guided solely along the RF path. Besides, due to the rectangular waveguide involved in the packaging structure, the DC block on the RF path in order to prevent the DC bias from being brought to the following components can be avoided. By taking into account the feeding network as well as the transition, the bias-tee is realized using CPWs based on a high-resistivity silicon ( $\mathrm{Si}$ ) substrate which has a thickness of $280 \mu \mathrm{m}$. The $\epsilon_{r}$ and $\tan \delta$ of the Si substrate are 11.6 and 4e-3, respectively [19].

The RF path consists of a CPW through line with a length of $1 \mathrm{~mm}$. The characteristic impedance is $50 \Omega$. For easier integration with the feeding network, the signal trace is designed to have an identical width which is $50 \mu \mathrm{m}$. The DC/IF path is connected to the RF path in the middle and the width of the signal trace is optimized to $20 \mu \mathrm{m}$. In both cases, the gap between the signal and ground traces is $30 \mu \mathrm{m}$. In order to prevent the RF signal from leaking to the DC/IF path, two open-circuited CPW stubs are implemented in parallel. Fig. 5 demonstrates the equivalent schematics of the proposed 


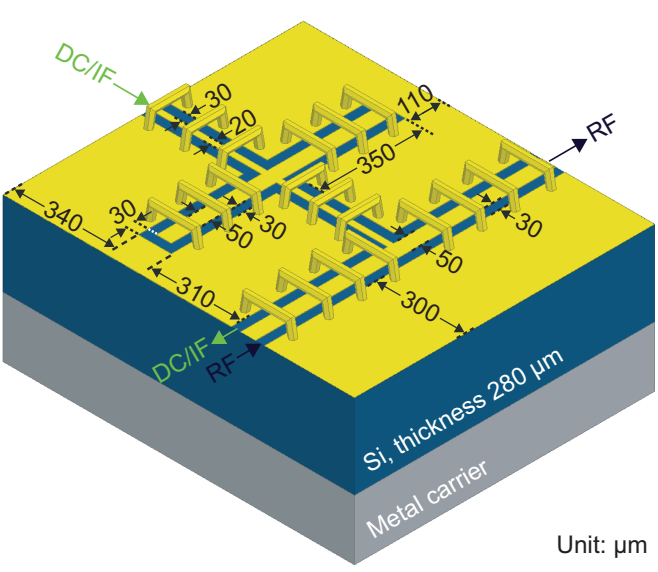

Fig. 4. Bias-Tee based on Si substrate.
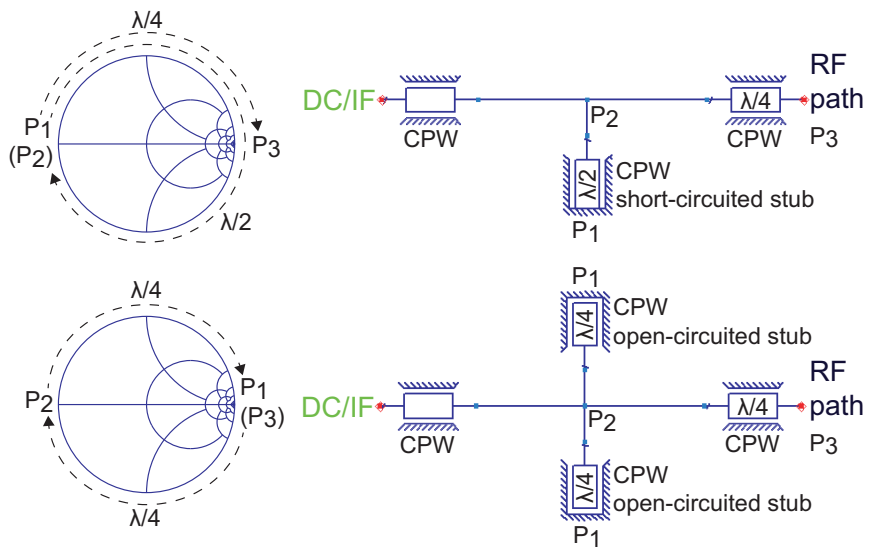

Fig. 5. Smith charts and the equivalent schematics of the bias-tee.

bias-tee and the corresponding impedance transformations on the Smith chart. The original idea for designing the bias-tee is to implement a through line structure on the RF path and a quarter-wavelength transformer on the DC/IF path which converts the termination from short to open. In this case, a quarter-wavelength $\mathrm{CPW}$ is followed by a half-wavelength CPW short-circuited stub. Though the RF signal can be isolated from the DC/IF path, the DC bias is directly shortcircuited by the stub. As an alternative, the half-wavelength CPW short-circuited stub is replaced by a quarter-wavelength CPW open-circuited stub. In addition, another identical opencircuited stub is added in parallel which further improves the isolation for the RF signal and at the same time, it does not increase the occupation area of the bias-tee.

Based on the second schematic shown in Fig. 5, the biastee is designed and the dimensions are optimized for the RF signal at W-band. For restricting parasitic modes, wire bonding bridges on the top of the substrate are also added along the paths. In the simulation, lumped ports with vertical perfect electric conductor (PEC) bridges are used as the excitation scheme. Fig. 6 shows the simulation results of the proposed bias-tee based on Si substrate with wire bonding bridges. The return loss is better than $16.6 \mathrm{~dB}$ with an associated insertion loss of $0.9 \mathrm{~dB}$. Besides, the simulated isolation for the $\mathrm{RF}$ signal is more than $18.6 \mathrm{~dB}$. In contrast, for the situation

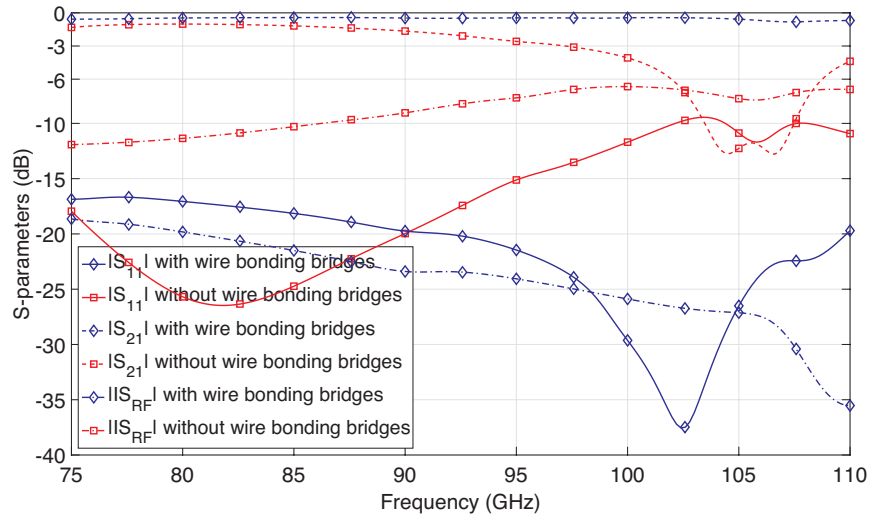

Fig. 6. Simulation results of the bias-tee based on Si substrate at W-band.

when wire bonding bridges are removed, the performance of the bias-tee degrades dramatically due to parasitic modes.

\section{Rectangular Waveguide-to-CPW Transition}

With an interface of rectangular waveguide, the RF signal generated by the photoconductor can be easily connected to other devices at the output of the photodetector. Since both the feeding network and the bias-tee are based on CPWs, the transition becomes an essential component in the packaging structure. Several different types of rectangular waveguide-toCPW transitions can be found in the literature [20]-[25] while E-plane probe exhibits noticeable performances especially at millimeter-wave and submillimeter-wave frequencies [26][29]. Fig. 7 shows the proposed transition at W-band using Eplane probe. A rectangular patch patterned on a high-resistivity $\mathrm{Si}$ substrate works as the E-plane probe which is inserted into the WR-10 rectangular waveguide through an aperture cut in the center of the broadwall parallel to the longitudinal axis. The E-plane probe is placed $740 \mu \mathrm{m}$ from the end of the rectangular waveguide which forms a quarter-wavelength transformer converting the termination from short to open. In order to guide the RF signal from the bias-tee to the E-plane probe smoothly, a CPW is included in the transition structure which has a length of $1 \mathrm{~mm}$ and a characteristic impedance of $50 \Omega$. Though the CPW is patterned on the same substrate, its dimensions are optimized for the new environment conditions due to the metal cover around the transition. In addition, efforts are put to restrict parasitic modes by adding wire bonding bridges, reducing the width of the substrate as well as the height of the cavity. As a result, the width of the signal trace is $80 \mu \mathrm{m}$ and the width of the ground traces is $110 \mu \mathrm{m}$ with a gap of $50 \mu \mathrm{m}$. For easier integration with the bias-tee, the CPW is tapered at the beginning.

In the simulation, the length of the rectangular waveguide is set to be $10 \mathrm{~mm}$ and wave ports are used as the excitation scheme. The rounded corners at the end of the rectangular waveguide are also included for being compatible with the milling process which results in a radius of $250 \mu \mathrm{m}$. Fig. 8 shows the simulation results of the proposed E-plane probe transition at W-band. With wire bonding bridges, the E-plane probe transition based on $\mathrm{Si}$ substrate exhibits a wideband 


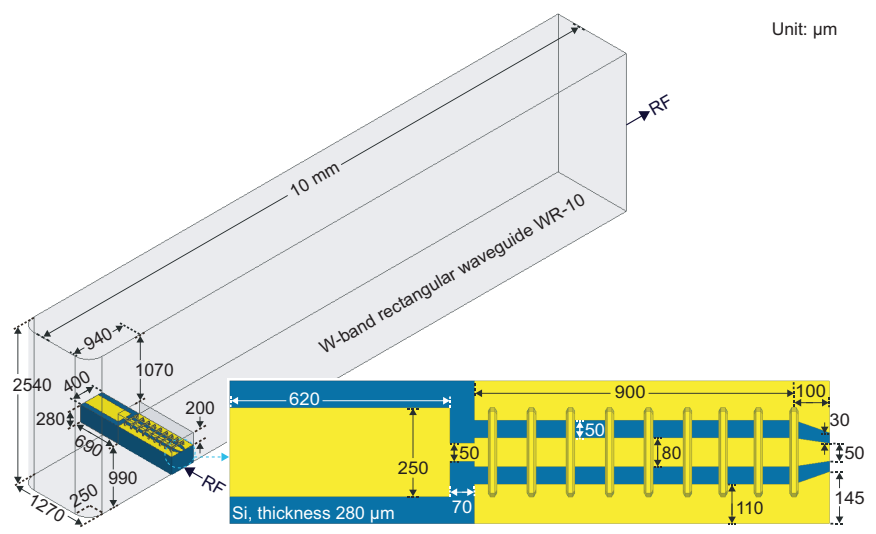

Fig. 7. Rectangular waveguide-to-CPW transition using E-plane probe.

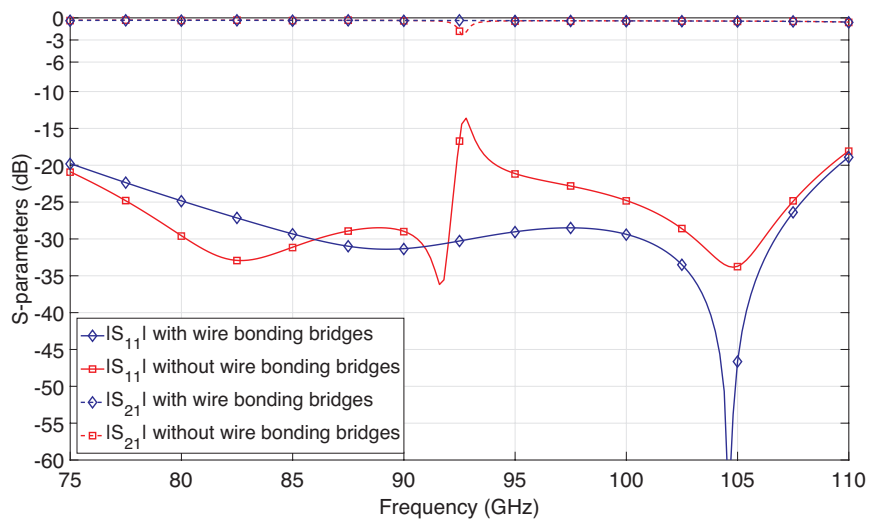

Fig. 8. Simulation results of the rectangular waveguide-to-CPW transition at W-band using E-plane probe.

behavior. The return loss remains better than $18.8 \mathrm{~dB}$ with an associated insertion loss of $0.6 \mathrm{~dB}$. In contrast, for the E-plane probe transition without wire bonding bridges, a notch can be observed around $93 \mathrm{GHz}$ which is due to the parasitic mode in the transition cavity.

\section{Chip-level Connection and Integration}

Based on the system diagram shown in Fig. 1, the individual components are integrated and chip-level connections are implemented. Fig. 9 demonstrates the proposed approach for integrating the feeding network, bias-tee, and E-plane probe transition. A metal carrier is used for realizing the WR-10 rectangular waveguide, packaging the E-plane probe transition, and providing physical support for other components. By milling the metal carrier into several platforms, the thickness difference between the $\mathrm{InP}$ and $\mathrm{Si}$ substrates can be compensated. As a result, the components are aligned by their top surfaces so that the vertical gap is avoided at the connections. In addition, wire bondings with a diameter of $25 \mu \mathrm{m}$ are used for not only chip-level connections but also providing ground connections to the components at different positions on the substrates. Since the wire bonding connections introduce parasitic inductance especially at high frequencies, the components are placed as close as possible and the height of the wire bondings is kept around $50 \mu \mathrm{m}$.

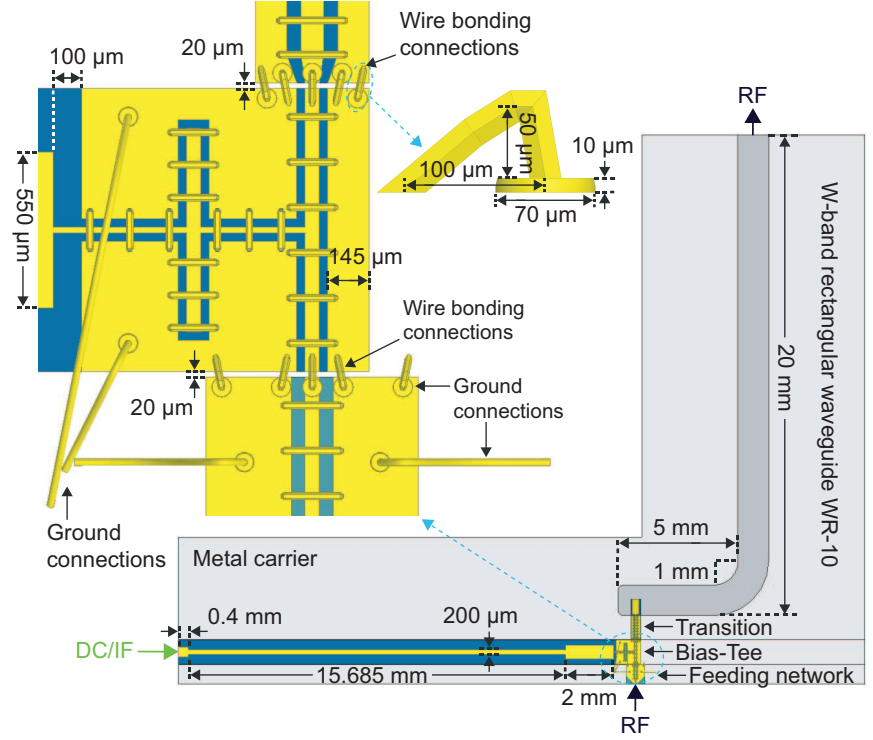

Fig. 9. Integration of the feeding network, bias-tee, and transition.

The bias-tee is optimized from the original design for an easier integration. On the RF path, by reducing the width from $300 \mu \mathrm{m}$ to $145 \mu \mathrm{m}$, the ground trace touches the metal carrier and it is aligned to the edge of the transition at the same time. By taking into account the DC bias as well as the potential IF signal, the signal trace is extended in order to reach the inputs which are connected to the bias-tee through a SMA connector at the far end. With the purpose of enlarging the bandwidth of the potential IF signal, an impedance transformer based on microstrip line is inserted to the DC/IF path whose width and length are $550 \mu \mathrm{m}$ and $2 \mathrm{~mm}$, respectively. After that, a microstrip line with a characteristic impedance of $50 \Omega$ is implemented for guiding the potential IF signal. The width is $200 \mu \mathrm{m}$ and the length is $15.685 \mathrm{~mm}$. At the end of the DC/IF path, there is a square pad with a length of $400 \mu \mathrm{m}$ which is large enough for connecting to the SMA connector. In order to be compatible with the experimental setup at W-band, the WR-10 rectangular waveguide is bent $90^{\circ}$ with a total length of approximately $25 \mathrm{~mm}$. For minimizing the reflection, the quadrant structure has an inner radius of $1 \mathrm{~mm}$ and it is placed $4 \mathrm{~mm}$ from the end of the rectangular waveguide.

In the simulation, different types of excitation schemes are used. A lumped port is assigned to a surface located in the gap between the signal trace and the ground pad on the feeding network. It represents the photoconductor and is used as the input of the RF signal as well as the output of the IF signal. A wave port is assigned to the outer surface of the rectangular waveguide which is the output of the RF signal. As for the input of the IF signal, another lumped port is assigned to the surface between the square pad on the bias-tee and the bottom metal carrier. The simulation results of the integrated components for both RF and IF signals are shown in Fig. 10. Since the RF signal operates at W-band, the simulation results range from $75 \mathrm{GHz}$ to $110 \mathrm{GHz}$. The return loss remains better than $15 \mathrm{~dB}$ with an associated insertion loss of $2.3 \mathrm{~dB}$ and the isolation is more than $16 \mathrm{~dB}$. However, the IF signal is 


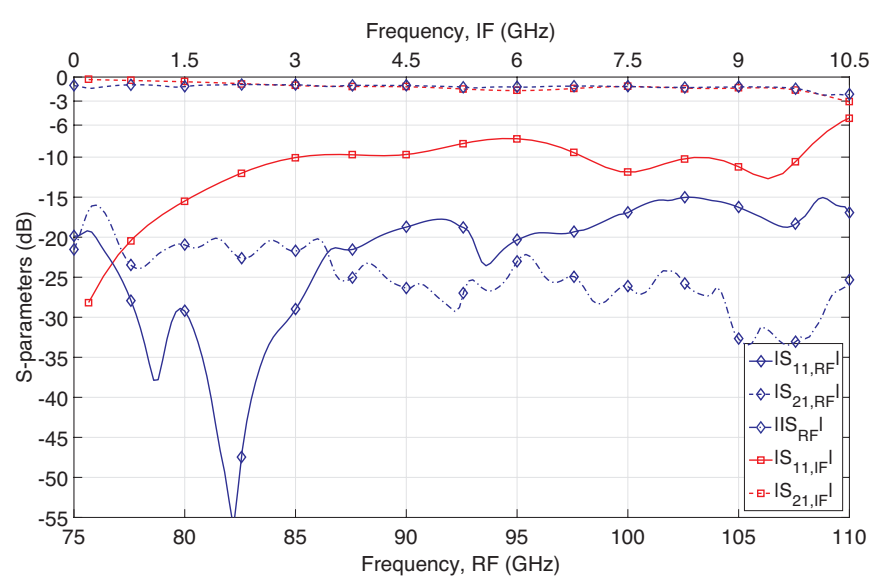

Fig. 10. Simulation results of the integrated components.

simulated up to $10.5 \mathrm{GHz}$. It achieves a return loss of $10 \mathrm{~dB}$ and an associated insertion loss of $1 \mathrm{~dB}$ up to $3 \mathrm{GHz}$ while they reach $7.7 \mathrm{~dB}$ and $2 \mathrm{~dB}$, respectively, at $10 \mathrm{GHz}$.

\section{E. Fabrication and Experimental Results}

In order to prove the designs and validate the proposed integration approach, the bias-tee, E-plane probe transition, and WR-10 rectangular waveguide demonstrated in Fig. 9 were fabricated. The bias-tee and E-plane probe transition were patterned on an n-type high-resistivity $\mathrm{Si}$ wafer which has a diameter of $50.8 \mathrm{~mm}$ and a crystal orientation of $<100>$. At the beginning, a conductor layer was deposited on the top of the Si wafer with a thickness of $200 \mathrm{~nm}$ using gold evaporation. After that, different layouts were patterned by following the process of photolithography. The wafer was diced into samples which were integrated into two identical prototypes at the end. Fig. 11 shows the integration of the fabricated bias-tee and E-plane probe transition. The metal package including the rectangular waveguide was divided into two parts and each part was fabricated using a brass block by milling process which was carried out by using a spinner with a radius of $250 \mu \mathrm{m}$. For accurate assembly, guide pins as well as screws were used and a standard WR-10 rectangular waveguide flange with annular recess was also applied to the fabricated brass package. The bias-tee and E-plane probe transition were first aligned on the brass carrier and the gaps were filled with silver epoxy. Then the wire bonding bridges along the CPWs, wire bonding connections between the components, and ground connections at different positions were added by ball bonding process. The brass cover was assembled at the last step.

Fig. 12 illustrates the experimental setup for on-wafer measurement at W-band as well as the device under test (DUT). The experimental setup comprises an Anritsu ME7808B vector network analyzer (VNA), two WR-10 extenders from Anritsu, and two ground-signal-ground (GSG) probes with a pitch size of $125 \mu \mathrm{m}$. The DUT has a back-to-back configuration which consists of two identical prototypes connecting to each other through the rectangular waveguide flanges.

By applying the GSG probes to the integrated bias-tees
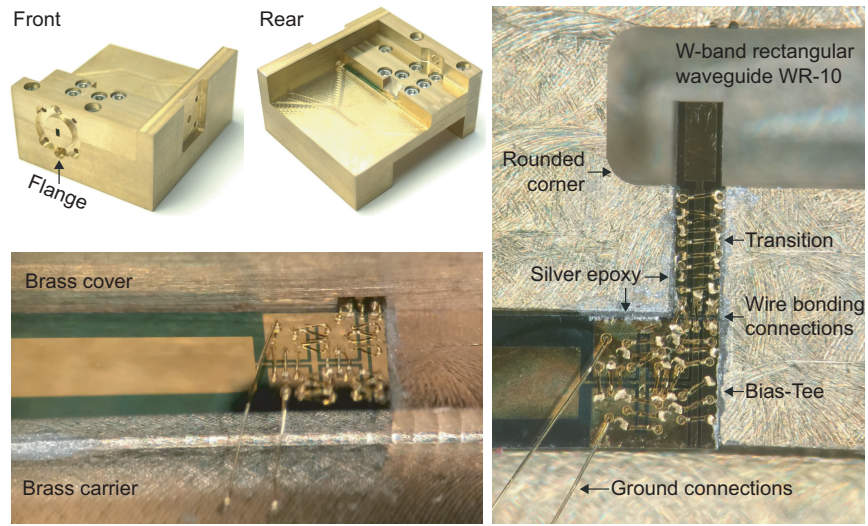

Fig. 11. Integration of the fabricated bias-tee and transition.

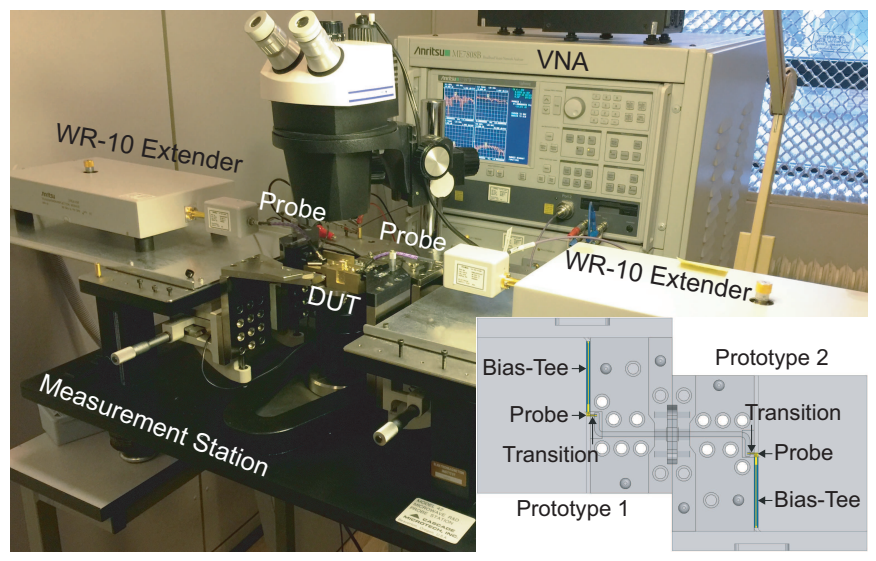

Fig. 12. Experimental setup for on-wafer measurement at W-band.

of the prototypes, two-port S-parameters were measured at W-band and on-wafer calibration was executed before the measurement. Fig. 13 shows both the measurement and the simulation results of the integrated bias-tee and transition in a back-to-back configuration. From $75 \mathrm{GHz}$ to $110 \mathrm{GHz}$, the measured return loss remains better than $9.4 \mathrm{~dB}$ with an associated insertion loss of $8.6 \mathrm{~dB}$. As a result, each prototype introduces less than $4.3 \mathrm{~dB}$ insertion loss at Wband. Compared with the simulation results, an extra insertion loss of $2.7 \mathrm{~dB}$ is achieved by each prototype which is mainly caused by the integration process especially the wire bonding connections between the components and the $\tan \delta$ variation of the Si substrate at such high frequencies. From the system integration point of view, a measured return loss of $9.4 \mathrm{~dB}$ is acceptable while the insertion loss can be compensated to some extend by the cascaded W-band amplifiers in the communication system.

\section{SYSTEM INTEGRATION}

\section{A. Fully-Packaged Photodetector}

Though the integration of the feeding network, bias-tee, and E-plane probe transition has been addressed, the photoconductor as well as other supplementary components such as optical fibers, connectors, and outer metal covers are also necessary in order to have a fully-packaged photodetector. At telecom 


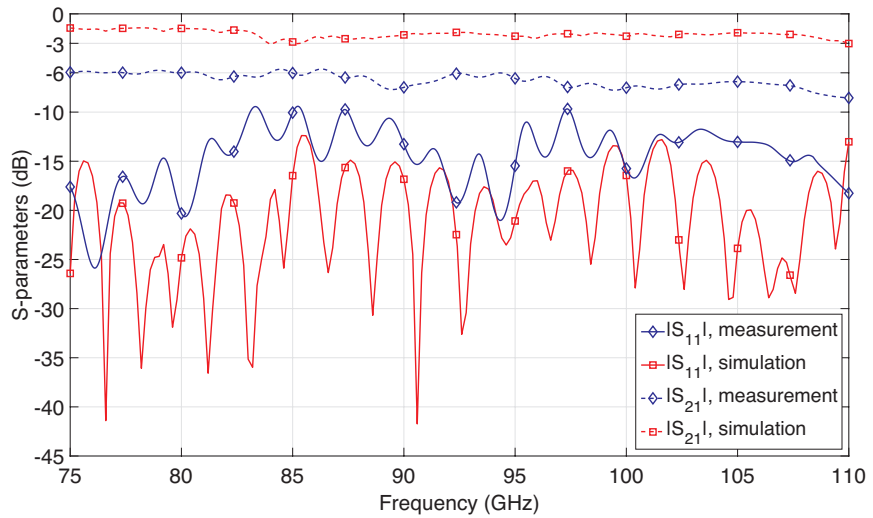

Fig. 13. Measurement results of the integrated bias-tee and transition at Wband in a back-to-back configuration.

wavelengths, the photoconductor is challenged by finding suitable photoconductive materials with a short carrier lifetime. Due to the low dark resistance, the DC bias that can be applied to the photoconductor is normally limited which makes it difficult to achieve a sufficient output power. According to [8], by combining erbium arsenide (ErAs), indium gallium arsenide (InGaAs), and indium aluminium arsenide (InAlAs) layers, the fabricated photoconductor exhibits excellent $\mathrm{THz}$ performances with a high dark resistance under continuous wave $(\mathrm{CW})$ operations. As a result, the $\operatorname{ErAs}: \operatorname{In}(\mathrm{Al}) \mathrm{GaAs}$ photoconductor is packaged into a $\mathrm{THz}$ photodetector which is used as a part of the transmitter in the communication system.

Fig. 14 illustrates the assembly of the fully-packaged photodetector. Apart from the principal components, a SMA connector inserting through the sidewall of the brass package was connected to the DC/IF path on the bias-tee and an angled physical contact (APC) connector with an optical fiber was used for guiding the optical signal all the way to the photoconductor. Since the optical signal needs to be illuminated to the photoconductor from the top, by taking into account the efficiency as well as accuracy, a fiber holder with customized dimensions was employed. The fiber holder was fabricated using a polyvinyl chloride (PVC) block by milling process and the optical fiber was fixed inside a though hole drilled from the top. Besides, the outer metal covers made from brass plates were added so that a fully-packaged device is set up with both physical and electrical protections.

\section{B. Experimental Setup and Results}

In order to validate the proposed system integration and packaging approach of the photodetector, the $\mathrm{THz}$ heterodyne communication system demonstrated in Fig. 15 is implemented. On the transmitter (Tx) side, a CW laser operating at $1550 \mathrm{~nm}$ is used as the light source and a polarization controller (PC) is added before the optical signal reaches the MZM where a RF signal at local oscillator frequency $\left(f_{L O}\right)$ is modulated to the optical signal achieving two optical tones with the expected distance $\left(2 f_{L O}\right)$ in the spectrum. Then an erbium-doped fiber amplifier (EDFA) is employed to enlarge the power of the optical signal and followed by an arrayed waveguide grating (AWG) which demultiplexes the two optical

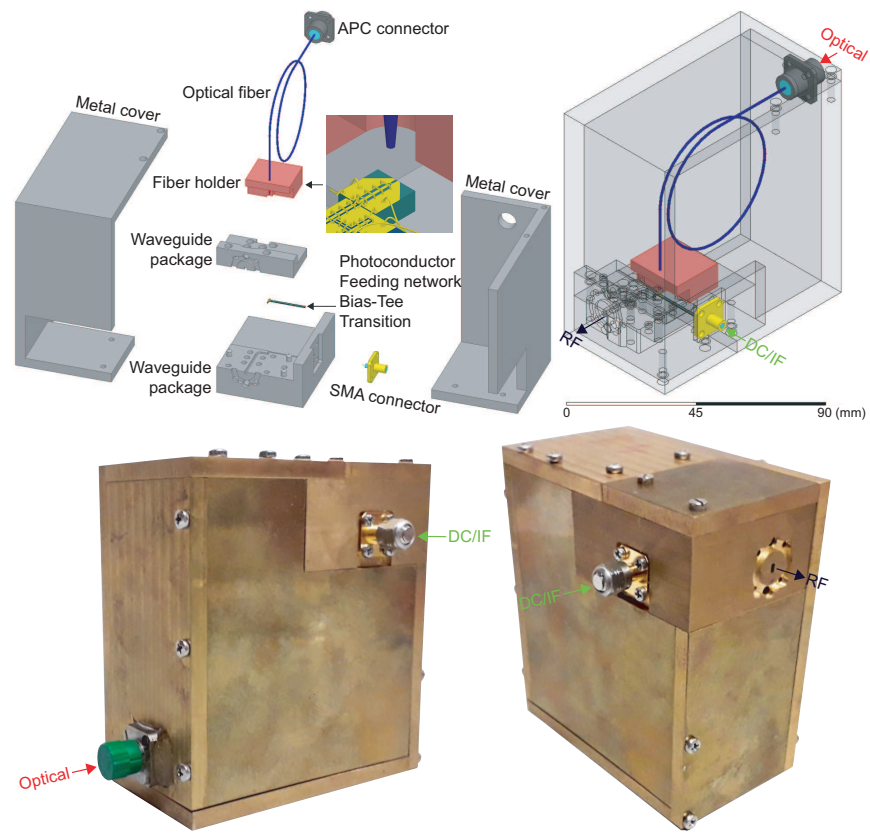

Fig. 14. Assembly of the fully-packaged photodetector.

tones. The data is modulated to one of the tones by using another MZM and a variable optical attenuator (VOA) is applied on the other tone which adjusts the power of the optical signal. After that, the two optical tones are coupled together and the spectrum of the optical signal is also presented. Another EDFA pushes up the power of the optical signal to $30 \mathrm{~mW}$ before being transmitted to the photodetector. The RF signal with the modulated data has a frequency component of $2 f_{L O}$ which is generated by the photodetector and then guided to a low noise amplifier (LNA) followed by a medium power amplifier (MPA). The W-band LNA and MPA from Radiometer Physics $\mathrm{GmbH}$ can provide gains of $40 \mathrm{~dB}$ and $10 \mathrm{~dB}$, respectively. For radiating the RF signal into free space, a W-band standard horn antenna is used which has a gain of $21 \mathrm{dBi}$.

On the receiver $(\mathrm{Rx})$ side, a comb fiber laser operating at $1550 \mathrm{~nm}$ is used as the light source and the repetition rate $\left(f_{\text {comb }}\right)$ is $40 \mathrm{GHz}$. The generated optical frequency comb goes through a wavelength selective switch (WSS) which reserves the two optical tones with a distance of $80 \mathrm{GHz}\left(2 f_{\text {comb }}\right)$ in the spectrum and the rest of the tones are suppressed. The power of the filtered optical signal is increased to 30 $\mathrm{mW}$ by using an EDFA and the spectrum is also shown in Fig. 15. The photodetector is based on an ErAs:InGaAs photoconductor which is attached to a log-periodic antenna and packaged using a hemispherical silicon lens. Since the DC bias is not required by the photoconductor in this case, the InAlAs layer is excluded, which according to [8] provides a higher mobility and a better sensitivity compared with the photoconductor used on the Tx side. When the IF signal is extracted by the photodetector, it has a frequency component of $\left|2 f_{\text {comb }}-2 f_{L O}\right|$ which can be tuned by sweeping the $f_{L O}$ on the Tx side. Besides, a transimpedance amplifier (TIA) and two electrical amplifiers (EAs) are employed which provide 


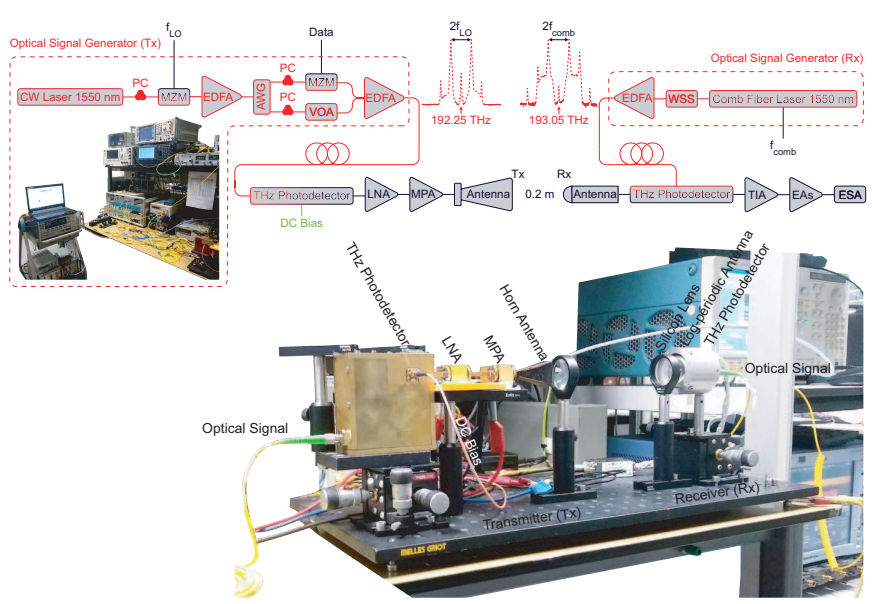

Fig. 15. Experimental setup of the $\mathrm{THz}$ heterodyne communication system.

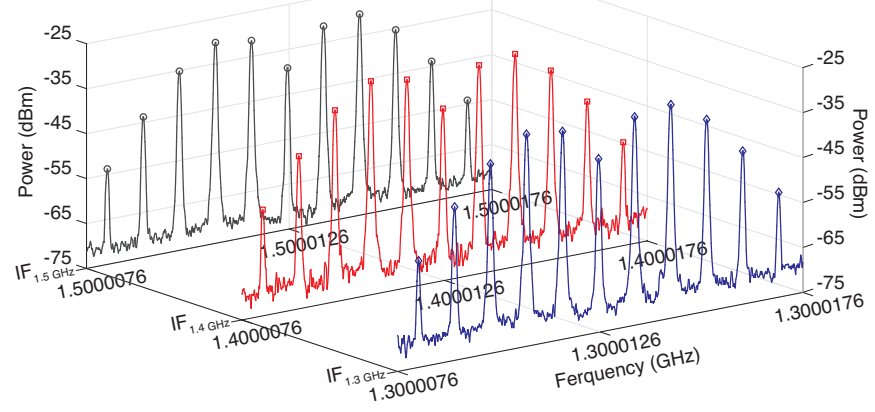

Fig. 16. Measurement results of the $\mathrm{THz}$ heterodyne communication system

gains of $20 \mathrm{~dB}, 20 \mathrm{~dB}$, and $17 \mathrm{~dB}$, respectively. Then the power of the IF signal is measured by using a Rohde \& Schwarz FSQ40 electrical spectrum analyzer (ESA).

Since the bandwidth of the communication system is limited to $2 \mathrm{GHz}$ due to the TIA on the $\mathrm{Rx}$ side, the IF signal is set to operate at $1.3 \mathrm{GHz}, 1.4 \mathrm{GHz}$, and $1.5 \mathrm{GHz}$ which correspond to $78.7 \mathrm{GHz}, 78.6 \mathrm{GHz}$, and $78.5 \mathrm{GHz}$, respectively, for the transmitted RF signals. The measurement results at different operating frequencies are compared in Fig. 16. Instead of a single frequency component, the measured IF signal exhibits several tones in the spectrum which might be due to a beating interference on the Tx side. In addition, the total powers of the IF signal at different operating frequencies are $-2.75 \mathrm{dBm},-2.78 \mathrm{dBm}$, and $-5.43 \mathrm{dBm}$, respectively, which are calculated by integrating the power over the corresponding frequency ranges read out from the ESA. Though the IF signal spreads slightly in the spectrum, the recovered tones achieve sufficient stability as well as power for supporting data transmissions with different modulation schemes. As a consequence, it validates the proposed system integration and packaging approach of the $\mathrm{THz}$ photodetector.

\section{CONCLUSiON}

In this work, the system integration and packaging of a photodetector at W-band for $\mathrm{THz}$ communications have been presented. Though the fully-packaged $\mathrm{THz}$ photodetector is based on an ErAs: $\operatorname{In}(\mathrm{Al}) \mathrm{GaAs}$ photoconductor, the proposed system integration and packaging approach can also be applied to other photoconductors as well as photodiodes. As the principal components involved in the integration, the feeding network, bias-tee, and transition have been designed, fabricated, and integrated. However, changing of environment conditions due to the packaging structure results in parasitic modes on the individual components. Wire bonding bridges were added along the planar transmission lines and it turned out to be effective in restricting parasitic modes. By implementing a CPW, the feeding network based on semi-insulating InP substrate supports low-loss transmissions from DC to 110 $\mathrm{GHz}$. For the bias-tee at W-band based on high-resistivity Si substrate, the RF path was realized using a CPW through line while the DC/IF path was extended in order to reach the inputs at the far end. The E-plane probe transition between the CPW and the WR-10 rectangular waveguide has been illustrated and it exhibits a wideband behavior. The proposed bias-tee and the E-plane probe transition including the WR-10 rectangular waveguide were integrated and measured on-wafer by connecting two prototypes in a back-to-back configuration. The wire bondings were used not only for chip-level connections but also for providing ground connections to the components at different positions. The assembly of the fully-packaged photodetector and the fabricated packaging structure have been demonstrated. A $\mathrm{THz}$ heterodyne communication system has been implemented in which the photodetector is involved on the Tx side as a fully-packaged device which can be used for other communication systems as well. Though the measured IF signal spreads in the spectrum, the stability as well as power of the recovered tones are sufficient for supporting data transmissions with different modulation schemes.

\section{ACKNOWLEDGMENT}

The authors would like to thank S. Schober and T. Schildknecht at the Department of Electrical Engineering and Information Technology, Technische Universität Darmstadt for assisting the fabrication processes.

\section{REFERENCES}

[1] T. Umezawa et al., "Bias-free operational UTC-PD above $110 \mathrm{GHz}$ and its application to high baud rate fixed-fiber communication and W-band photonic wireless communication," J. Light. Technol., vol. 34, no. 13, pp. 3138-3147, July. 2016.

[2] L C. P. Cavalcante et al., "Performance evaluation of wavelet-coded OFDM on a $4.9 \mathrm{~Gb} / \mathrm{s}$ W-band radio-over-fiber link," J. Light. Technol., vol. 35, no. 14, pp. 2803-2809, July. 2017.

[3] A. Bekkali et al., "Real-time $10 \mathrm{GbE}$ data transmission over a converged RoF links and 96-GHz wireless bridge," IEEE Photon. Technol. Lett., vol. 29, no. 1, pp. 15-18, Jan. 2017.

[4] A. Morales, R. Puerta, S. Rommel, and I. T. Monroy, "1 Gb/s chaotic encoded W-band wireless transmission for physical layer data confidentiality in radio-over-fiber systems," Opt. Express, vol. 26, no. 17, pp. 22296 22306, Aug. 2018.

[5] A. Beling and J. C. Campbell, "High-speed photodiodes," IEEE J. Sel. Topics Quantum Electron., vol. 20, no. 6, pp. 57-63, Nov. 2014.

[6] S. Jones et al., "Ultra-wideband tunable dual-mode laser for continuous wave terahertz generation," J. Light. Technol., vol. 32, no. 20, pp. 34613467, Oct. 2014.

[7] S. Preu et al., "1550 nm ErAs:In(Al)GaAs large area photoconductive emitters," Appl. Phys. Lett., vol. 101, no. 10, pp. 11051-11054, Sept. 2012 . 
[8] A. D. J. Fernandez Olvera, H. Lu, A. C. Gossard, and S. Preu, "Continuous-wave $1550 \mathrm{~nm}$ operated terahertz system using ErAs: $\operatorname{In}(\mathrm{Al}) \mathrm{GaAs}$ photoconductors with $52 \mathrm{~dB}$ dynamic range at 1 THz," Opt. Express, vol. 25, no. 23, pp. 29492-29500, Nov. 2017.

[9] U. Nandi, J. C. Norman, A. C. Gossard, H. Lu, and S. Preu, "550$\mathrm{nm}$ driven ErAs: $\mathrm{In}(\mathrm{Al}) \mathrm{GaAs}$ photoconductor-based terahertz time domain system with $6.5 \mathrm{THz}$ bandwidth," J. Infrared Millim. Terahertz Waves, vol. 39, no. 4, pp. 340-348, Apr. 2018.

[10] C. Jiang, V. Krozer, H. G. Bach, G. G. Mekonnen, and T. K. Johansen, "Broadband packaging of photodetectors for $100 \mathrm{~Gb} / \mathrm{s}$ Ethernet applications," IEEE Trans. Compon. Packag. Manuf. Technol., vol. 3, no. 3, pp. 422-429, Mar. 2013.

[11] H. G. Bach et al., "InP-based waveguide-integrated photodetector with 100-GHz bandwidth," IEEE J. Sel. Topics Quantum Electron., vol. 10, no. 4 , pp. 668-672, July. 2004.

[12] G. G. Mekonnen et al., "InP waveguide-integrated pin-photodiode hybrid packaged with an HBT-DEMUX-chip for receiver modules of 80-100 $\mathrm{Gb} / \mathrm{s}$ data rates," in proc. 34th Eur. Conf. Opt. Commun. (ECOC), Sept. 2008, pp. 1-2.

[13] C. Jiang, V. Krozer, H. G. Bach, G. G. Mekonnen, and T. K. Johansen, "Packaging of photodetector modules for $100 \mathrm{Gbit} / \mathrm{s}$ applications using electromagnetic simulations," in proc. 39th Eur. Microw. Conf. (EuMC), Sept. 2009, pp. 1369-1372.

[14] H. G. Bach et al., "Novel $107 \mathrm{~Gb} / \mathrm{s}$ bias-feeding photodetector OEIC for efficient low-cost photoreceiver co-packaging," in proc. 35th Eur. Conf. Opt. Commun. (ECOC), Sept. 2009, pp. 1-2.

[15] A. Stöhr et al., "Millimeter-wave photonic components for broadband wireless systems.," IEEE Trans. Microw. Theory Techn., vol. .58, no. 11 pp. 3071-3082, Nov. 2010.

[16] M. Jarrahi, "Advanced photoconductive terahertz optoelectronics based on nano-antennas and nano-plasmonic light concentrators," IEEE Trans. THz Sci. Technol., vol. 5, no. 3, pp. 391-397, May. 2014.

[17] I. Flammia et al., "71-76 GHz grounded-coplanar-waveguide-torectangular-waveguide transition with integrated planar bias tee for quasihermetic radio-over-fiber wireless transmitter," in proc. 7th Eur. Microw. Integr. Circuits Conf. (EuMIC), Oct. 2012, pp. 512-515.

[18] S. Adachi, Physical Properties of III-V Semiconductor Compounds - InP, InAs, GaAs, GaP, InGaAs, and InGaAsP, John Wiley, 1992.

[19] D. M. Pozar, Microwave Engineering, John Wiley, 2012.

[20] M. Moallem, J. East, and K. Sarabandi, "A broadband, micromachined rectangular waveguide to cavity-backed coplanar waveguide transition using impedance-taper technique," IEEE Trans. THz Sci. Technol., vol. 64, no. 1 , pp. 49-55, Jan. 2014

[21] N. Vahabisani and M. Daneshmand, "Monolithic wafer-level rectangular waveguide and its transition to coplanar waveguide line using a simplified 3-D fabrication process," IEEE Trans. Compon. Packag. Manuf. Technol., vol. 4, no. 1, pp. 168-176, Jan. 2014.

[22] T. Tajima, H. J. Song, H. Matsuzaki, and M. Yaita, "LTCC-integrated $\mathrm{H}$-plane bends for THz antenna-in-package solution," IEEE Microw. Compon. Lett., vol. 27, no. 5, pp. 440-442, May. 2017.

[23] H. J. Song, H. Matsuzaki, and M. Yaita, "Sub-millimeter and terahertzwave packaging for large chip-width MMICs," IEEE Microw. Compon. Lett., vol. 26, no. 6, pp. 422-424, June. 2016.

[24] K. M. K. H. Leong et al., "A 340-380 GHz integrated CB-CPW-towaveguide transition for sub millimeter-wave MMIC packaging," IEEE Microw. Compon. Lett., vol. 19, no. 6, pp. 413-415, June. 2009.

[25] Y. Dong, T. K. Johansen, V. Zhurbenko, and P. J. Hanberg, "Rectangular waveguide-to-coplanar waveguide transitions at U-band using E-plane probe and wire bonding," in proc. 46th Eur. Microw. Conf. (EuMC), Oct. 2016, pp. 5-8.

[26] S. Jameson and E. Socher, "A wide-band CMOS to waveguide transition at mm-wave frequencies with wire-bonds," IEEE Trans. Microw. Theory Techn., vol. 63, no. 9, pp. 2741-2750, Sept. 2015.

[27] M. Vahidpour and K. Sarabandi, "2.5D micromachined $240 \mathrm{GHz}$ cavitybacked coplanar waveguide to rectangular waveguide transition," IEEE Trans. THz Sci. Technol., vol. 2, no. 3, pp. 315-322, May. 2012.

[28] T. Reck, R. M. Weikle, and N. S. Barker, "A waveguide to unenclosed coplanar waveguide transition," IEEE Trans. Microw. Theory Techn., vol. 58 , no. 9 , pp. $2420-2425$, Sept. 2010.

[29] K. Eriksson, P. J. Sobis, S. E. Gunnarsson, J. Hanning, and H. Zirath, "InP DHBT amplifier modules operating between 150-300 GHz using membrane technology," IEEE Trans. Microw. Theory Techn., vol. 63, no. 2, pp. 433-440, Feb. 2015

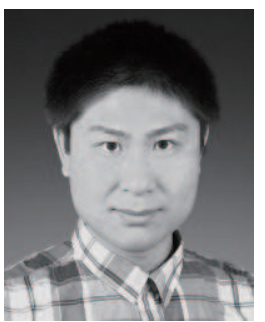

Yunfeng Dong ( $\left.S^{\prime} 17\right)$ received the B.Sc. degree in electronic information engineering from the Beijing University of Technology (BJUT), Beijing, China, in 2012, the M.Sc. and Ph.D. degrees in electrical engineering from the Technical University of Denmark (DTU), Lyngby, Denmark, in 2014 and 2019, respectively.

From September 2011 to July 2012, he was an exchange student with the South-Eastern Finland University of Applied Sciences (XAMK), Mikkeli, Finland. From October 2014 to December 2014, he was a research assistant with the Electromagnetic Systems Group (EMS), DTU, Lyngby, Denmark. From October 2017 to November 2017, he was a visiting scholar with the Terahertz Devices and Systems Group, the Technische Universität (TU) Darmstadt, Darmstadt, Germany. He is currently working as a Postdoc at EMS, DTU, Lyngby, Denmark. His research interests include developing innovative strategies that are suitable for hybrid integration and packaging at millimeter-wave and terahertz frequencies.

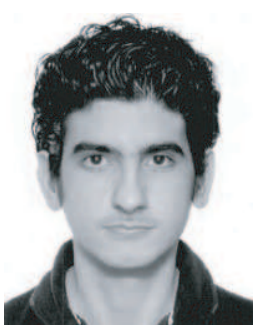

Anuar de Jesus Fernandez Olvera received the B.Sc. degree in electronic and computer engineering (summa cum laude) from the Tecnologico de Monterrey, Monterrey, Mexico, in 2010, the M.Sc. degree in electrical engineering (cum laude) from the Technische Universiteit Eindhoven (TU/e), Eindhoven, Netherlands, in 2015, and is currently working towards the Ph.D. degree at the Technische Universität (TU) Darmstadt, Darmstadt, Germany.

$\mathrm{He}$ is currently with the Terahertz Devices and Systems Group, TU Darmstadt, Darmstadt, Germany, under the framework of the EU H2020 project CELTA (Convergence of Electronics and Photonics Technologies for Enabling Terahertz Applications). As a part of the project, he had research stays at the Universidad Carlos III de Madrid (UC3M), Madrid, Spain and at Toptica Photonics AG, Gräfelfing, Germany. His current research interests include the development of broadband $\mathrm{THz}$ sources and detectors based on photomixing for different applications, including photonic vector analyzer, $\mathrm{THz}$ communication systems, and nondestructive testing.

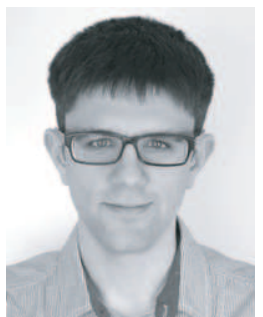

Alvaro Morales received the B.Sc. degree in telecommunications technologies engineering, the M.Sc. degree in telecommunications engineering from the University of Valladolid (UVa), Valladolid, Spain, in 2014 and 2016, respectively, and is currently working towards the Ph.D. degree at the Technische Universiteit Eindhoven (TU/e), Eindhoven, Netherlands.

From 2015 to 2017, he completed the thesis and worked as a research assistant with the MetroAccess and Short Range Communication Systems Group, the Technical University of Denmark (DTU), Lyngby, Denmark. $\mathrm{He}$ is currently with the Institute for Photonic Integration (IPI), TU/e, Eindhoven, Netherlands, under the framework of the EU H2020 project CELTA (Convergence of Electronics and Photonics Technologies for Enabling Terahertz Applications). His current research interests include millimeter-wave and sub-terahertz communications, radio-over-fiber systems, beamforming and photonic integrations. 


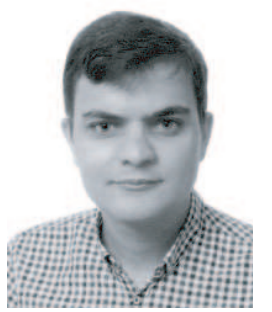

Mario Méndez Aller received the B.Sc. degree in telecommunications, the M.Sc. degree in multimedia and communications from the Universidad Carlos III de Madrid (UC3M), Madrid, Spain, in 2013 and 2014 , respectively, and is currently working towards the Ph.D. degree at the Technische Universität (TU) Darmstadt, Darmstadt, Germany.

$\mathrm{He}$ is currently with the Terahertz Devices and Systems Group, TU Darmstadt, Darmstadt, Germany. His current research interests include continuous wave terahertz photomixer sources, terahertz waveguides, and terahertz spectroscopy.

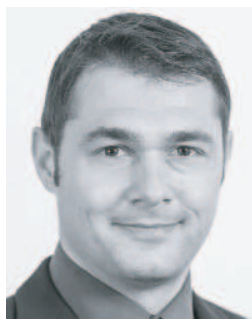

Sascha Preu received the Diploma and Ph.D. degrees in physics (summa cum laude) from the University of Erlangen-Nuremberg, Erlangen, Germany, in 2005 and 2009, respectively.

From 2004 to 2010, he was with the Max Planck Institute for the Science of Light, Erlangen, Germany. From 2010 to 2011, he was with the Materials and Physics Department, University of California, Santa Barbara, CA, USA. From 2011 to 2014, he was at the Chair of Applied Physics, University of Erlangen-Nuremberg. He is currently leading the Terahertz Devices and Systems Group, Department of Engineering and Information Technology, Technische Universität Darmstadt, Darmstadt, Germany. $\mathrm{He}$ has authored or co-authored more than 80 journal articles or conference contributions. His current research interests include the development of semiconductor-based terahertz sources and detectors, incuding photomixers, photoconductors, field effect transistor rectifiers, applications of terahertz radiation, in particular the characterization of novel terahertz optics, and materials such as whispering gallery mode resonators and graphene.

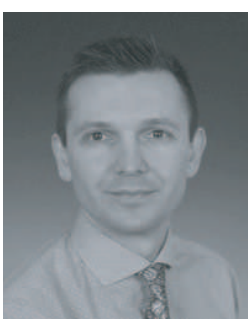

Vitaliy Zhurbenko (S'03-GSM'05-M'08) received the M.Sc. degree in electrical engineering from the Kharkiv National University of Radio Electronics, Kharkiv, Ukraine, in 2001, and the Ph.D. degree in electrical engineering from the Technical University of Denmark (DTU), Lyngby, Denmark, in 2008.

In 2005, he joined DTU, where he is currently an Associate Professor. His current research and teaching interests include microwave and millimeterwave sensing for biomedical and security applications; microwave and millimeter-wave components and integrated circuits for instrumentation applications; antennas and passive circuit design and characterization; and magnetic resonance technology.

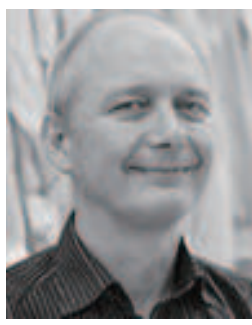

Peter Jesper Hanberg received the M.Sc. and Ph.D. degrees from the University of Copenhagen, Copenhagen, Denmark, in 1983 and 1987, respectively.

Since then he has worked over 30 years with semiconductor physics and processing gaining expertise in MOVPE and MBE epitaxial growth, thin film deposition techniques, wet and dry etching and a variety of characterization tools. After university he joined the Telecommunications Research Lab in Denmark where he set up a full III-V semiconductor cleanroom for fabricating high speed laser diodes. This work was continued in the company Giga later acquired by Intel. After this followed work on VCSELs and nanowire LEDs in the companies Alight and GLO. He has been employed at DTU Danchip since December 2010 as Process Generalist supporting customers of the cleanroom with device and process development, training on equipments and teaching courses on processing technology.

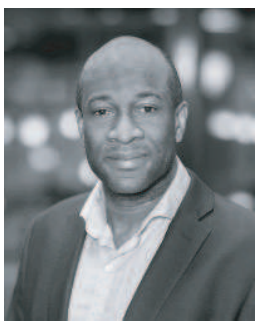

Chigo Okonkwo (M'09-SM'18) received the M.Sc. degree in telecommunications and information systems, and the Ph.D. degree in optical signal processing from the University of Essex, Essex, UK, in 2002 and 2009, respectively.

From 2009 to 2012, he was a Senior Research Fellow at the Technische Universiteit Eindhoven (TU/e), Eindhoven, Netherlands. He was also the Co-Investigator on a bilateral research framework project with South Korean organization ETRI on high-capacity coherent transmission systems. Since 2012, he is an Assistant Professor at the Institute for Photonics Integration (IPI), TU/e, where he is currently leading the high capacity optical transmission laboratory of the Electro-Optical Communications Group (EOC). His current research interests include scaling the number of transmitted fiber modes, development of few-mode as well as multi-core fibers and amplifiers, Space Division Multiplexing (SDM) focused on the feasibility of Petabit/s transmission in a single fiber. He serves as a reviewer for Nature Photonics and has (co)authored more than 200 publications in conferences and journals.

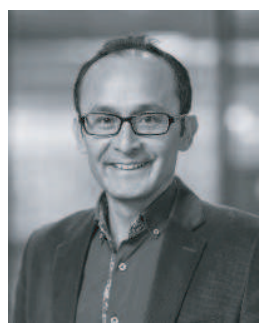

Idelfonso Tafur Monroy (M'94-SM'14) received the M.Sc. degree in multichannel telecommunication from the Bonch-Bruevich Institute of Communications, St. Petersburg, Russia, in 1992, and the Ph.D. degree in electrical engineering from the Technische Universiteit Eindhoven (TU/e), Eindhoven, Netherlands, in 1999.

From 1999 to 2006, he was with the ElectroOptical Communications Group (EOC), TU/e, Eindhoven, Netherlands, after which he became a Professor at the Department of Photonics Engineering, Technical University of Denmark (DTU), Lyngby, Denmark. In the meantime, he was also a Guest Professor at the Beijing University of Post and Telecommunications (BUPT), Beijing, China, Visiting Scientist at the University of California, Berkeley, CA, USA, and Fellowship Professor at ITMO University (Russia), St. Petersburg, Russia. Since July 2017, He is a Professor at the Photonic Terahertz Systems, TU/e, Eindhoven, Netherlands, and since November 2018, the director of the Photonic Integration Technology Center (PITC). His current research interests include photonics technologies for terahertz systems, converged electronic-photonic integrated circuits for applications in security, communications, sensing and computing. He is coauthor of over 500 journal and conference papers and has graduated $22 \mathrm{PhD}$ students.

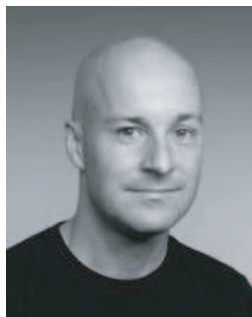

Tom Keinicke Johansen (S'04-M'04) received the M.Sc. and Ph.D. degrees in electrical engineering from the Technical University of Denmark (DTU), Lyngby, Denmark, in 1999 and 2003, respectively.

In 1999, he joined the Electromagnetic Systems Group (DTU Elektro), DTU, where he is currently an Associate Professor. From September 2001 to March 2002, he was a Visiting Scholar with the Center for Wireless Communication, University of San Diego, San Diego, CA, USA. He has spent several external research stays at the Ferdinand Braun Institute (FBH), Berlin, Germany. His research interests include the modeling of high-frequency solid-state devices, millimeter-wave and submillimeterwave integrated circuit design. 